\title{
Ergonomic risk assessment of nasogastric tube placement and implications for design and training
}

\author{
Oliver Anderson ${ }^{\mathrm{a}}$, Peter Buckle ${ }^{\mathrm{a}}$ and George Hanna ${ }^{\mathrm{a}}$ \\ ${ }^{a}$ Department of Surgery and Cancer, Imperial College London, UK
}

\begin{abstract}
The placement of nasogastric (NG) tubes is known to be prone to error and may lead to serious issues for patient safety and wellbeing. In some instances these have been catastrophic and resulted in the death of the patient. This study sought to explore the potential risks associated with this procedure through the use of systematic prospective risk assessment. The research team used the Prospective Hazard Analysis (PHA) toolkit developed by Ward et al (2010). The study has shown a wide number of risks that cover equipment design, work organisation, and training issues. The link between equipment design and training provides an important example of the need for a systematic approach to reducing errors and improving resilience in this aspect of healthcare.
\end{abstract}

Keywords: Nasogastric tubes, patient safety, prospective risk assessment

\section{Introduction}

Significant concerns exist re the correct placement of nasogastric (NG) tubes. These concerns relate to the potential for harm to patients when feeding is delivered via the tube if it is not in the correct location. In some instances, for example if the tube has inadvertently entered the lung, the results can be catastrophic for the patient. Incidences leading to the death of a patient are rare, yet regular, occurrences [5].

Approximately 1 million NG tubes are used every year in the English \& Welsh NHS. They are associated with the fatal preventable adverse event of misplaced NG tube feeding into the lungs. Despite measures to prevent this complication that is classified by the National Patient Safety Agency (NPSA) as a 'never event,' it continues to occur. Since 2005, 21 deaths and 79 cases of harm have been reported $[6,7]$.

\section{Method}

Members of the project team observed the process of checking the placement of NG tubes and created a process flow diagram. Failures were generated through reviews of the literature, observations, and interviewing patients and healthcare staff in focus groups with key stakeholders. This ensured that potential failures were detected through a triangulated approach. Individual patients and healthcare staff validated the process flow diagrams and rated the effects of each failure on three 4-point scales: frequency, severity and detectability.

i) System analysis: The study was based on a systems approach that sought to gain information on the use of NG tubes from a clearly identified set of users. To identify all these users and to establish the other components of the system that may impact on the efficacy of NG tube placement, a systems map was constructed. Such maps have been reported elsewhere in the ergonomics/patient safety literature $[2,3,4]$. Figure 1 shows the map developed in this study. This was constructed with input from a range of healthcare professionals, including the research team, involved with tube placement or other aspects of tube use. 
ii) Ethics: Ethical agreement for the study was granted from NHS research ethics committees (Refs: 10/H0706/45 \& 10/H0724/76).

iii) Selection of Risk Assessment Methodology: A recent research study has generated a new tool kit for healthcare use of Prospective Hazard analysis [7]. This was used as the basis for selecting an appropriate risk assessment approach, based on the characteristics of the healthcare system being assessed. Using this approach Failure modes effects analysis was selected as one appropriate method. Additional methods, e..g So-what-if (SWIFT) technique, may also have been desirable but time and other resources limited our research to one risk assessment approach.

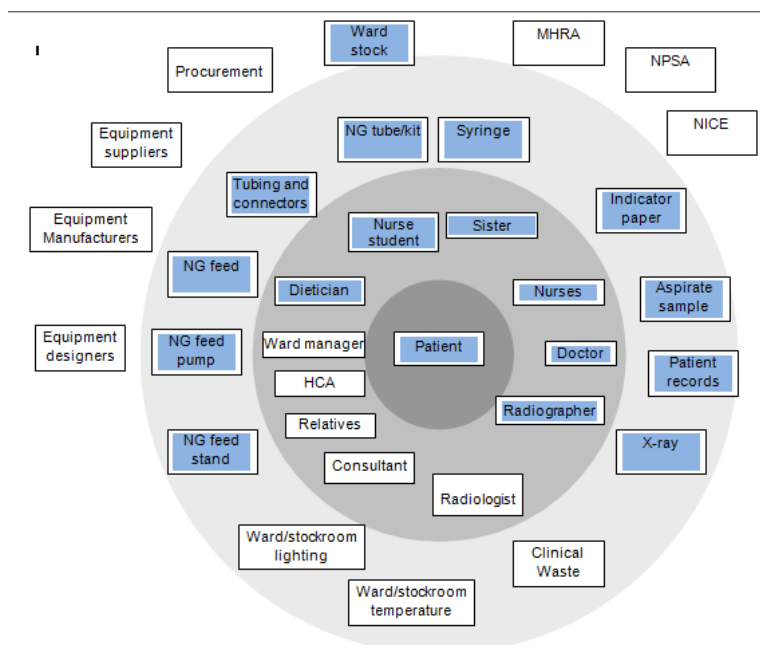

Figure 1 Systems Map of key components in NG tube use

iv) Task analysis: A simplified task analysis was undertaken. This was subsequently used to help with 'prompts' when undertaking focus groups and FMEA activities. The task analysis for one par tof the process is shown in figure 2 .

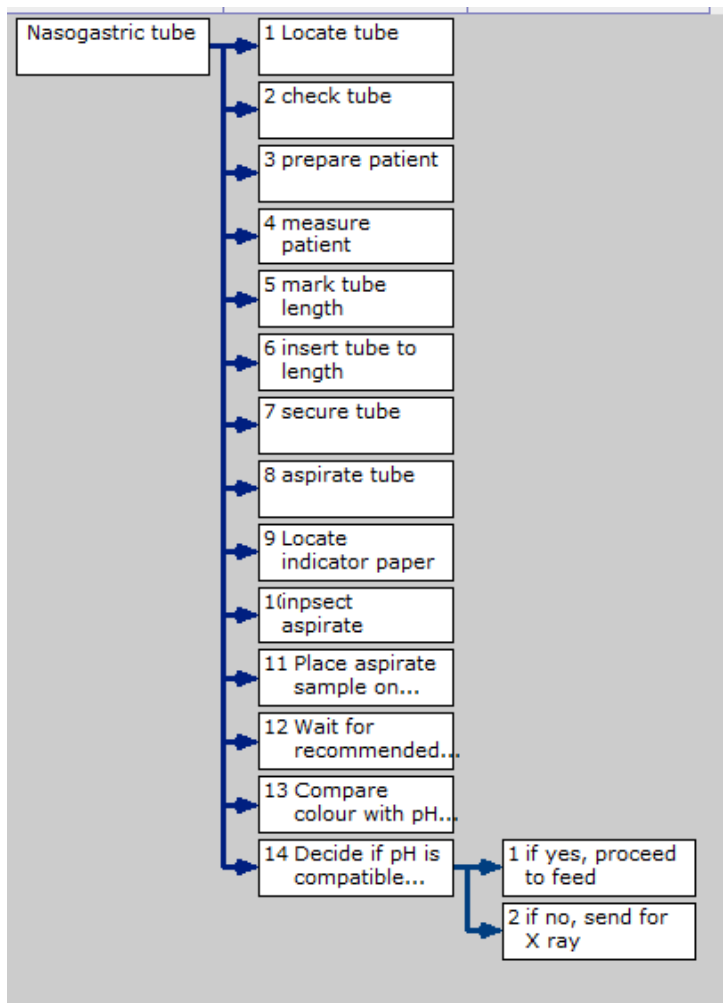

Figure 2 Example of task flow analysis for NG tube placement

\section{Results}

The process followed for undertaking the risk assessment was deemed to be successful. Participants were readily identified, were generally agreeable to participation and provided positive feedback on the experience and on the process. The work pressures of a modern NHS hospital meant that the time constraints on those consenting to be participants necessitated a concise approach to group discussion. Similarly, there was a need to undertake the FMEA exercise with individual staff.

The following table identifies a selection of the failure modes associated with this procedure. The full list with the accompanying risk scores is reported elsewhere [1]. 
Table 1

Examples of failure modes

\begin{tabular}{|c|c|}
\hline Process steps & Failure of omission \\
\hline Check NG tube protocol & Do not check NG protocol \\
\hline Find NG tube & No tube \\
\hline Find $\mathrm{pH}$ indicator paper & No $\mathrm{pH}$ paper \\
\hline Find syringe & No syringe \\
\hline Check tube & No check done \\
\hline Apply gel to tube & No gel applied \\
\hline Prepare patient & No patient \\
\hline Measure patient & No measurement \\
\hline Mark tube length & No tube mark \\
\hline Insert tube to length & Tube not inserted \\
\hline Secure tube & Tube not secured \\
\hline Aspirate tube & Tube not aspirated \\
\hline \multicolumn{2}{|c|}{ If no aspirate - roll patient and try agair Patient not rolled or re-aspirated } \\
\hline Inspect aspirate & \\
\hline \multicolumn{2}{|c|}{ Place aspirate sample on indicator paper No test of aspirate performed } \\
\hline Wait recommended time & Wait too long \\
\hline Compare colour with $\mathrm{pH}$ chart & Do not compare with chart \\
\hline Decide on $\mathrm{pH}$ value & Do not decide on $\mathrm{pH}$ value \\
\hline Document $\mathrm{pH}$ in (nursing) notes & Do not document \\
\hline Decide if tube can be used or needs an & Do not decide that whether tube \\
\hline $\mathrm{X}$-ray tube & Do not $x$-ray tube \\
\hline Interpret $\mathrm{X}$-ray & Do not interpret $\mathrm{x}$-ray \\
\hline Document $x$-ray result in the notes & Do not document \\
\hline Feed via tube & Do not feed via tube \\
\hline
\end{tabular}

\section{Discussion}

This paper has shown how an effective approach can be taken to mapping a system for NG tube placement. This map has formed the basis for the identification of key staff to be interviewed (as part of small focus groups.) This has enabled us to establish the difficulties and other issues they have encountered in the undertaking of NG tube placement. In particular, the participants have helped identify tasks that might compromise patient safety. The subsequent use of a prospective risk assessment methodology (FMEA) has enabled the researchers to quantify perceived risks and to use these as a basis for advancing alternative methods that minimise some of the important risks identified [1].

These alternative approaches include the development of a more reliable test, the identification of specific training needs, the need to assess risks in additional parts of the system (e.g. in areas where test materials are stored.)

The research has already demonstrated how knowledge and mental models of how and why the test is done and where it might fail vary substantially across healthcare professionals.

\section{References}

[1] Anderson, $\mathrm{O}$ et al Design, development and validation of a safer enzyme-based nasogastric tube position test Gastroenterology (under submission)

[2] Buckle, P Clarkson PJ, Coleman R, Bound, J , Ward J and Brown, J (2010) Systems mapping workshops and their role in understanding medication errors in healthcare Applied Ergonomics 41, 645-656

[3] Buckle P, Clarkson PJ, Coleman R, Ward J and Anderson J (2006) Patient safety, systems design and ergonomics Applied Ergonomics, 37, 491-500.

[4] Clarkson PJ, Buckle P, Coleman R, Stubbs D, Ward J, Jarrett J, Lane R, and Bound J (2004) Design for patient safety: a review of the effectiveness of design in the UK Health Service. Journal Engineering Design, 15, 123-140

[5] Coombes R. (2005) NHS safety agency issues guidance on nasogastric tubes $B M J$ 330(7489):438

[6] Lamont T, Beaumont C, Fayaz A, et al. (2011) Checking placement of nasogastric feeding tubes in adults (interpretation of $\mathrm{x}$ ray images): summary of a safety report from the National Patient Safety Agency. BMJ 342 :d2586

[7] Hanna GB, Phillips L, Priest O, et al. (2010) Improving the safety of nasogastric feeding tube insertion. Developing guidelines for the safe verification of feeding tube position - a decision analysis approach. A Report for the NHS Patient Safety Research Portfolio. July 2010. 2010

[8] Ward, J.R., Clarkson, P.J., Buckle, P., Berman, J., Lim, R. and Jun, G.T. (2010) 'Prospective hazard analysis: Tailoring prospective methods to a healthcare context', Patient Safety Research Portfolio, Report 